\title{
Algebraicity of Induced Riemannian Curvature Tensor on Lightlike Warped Product Manifolds
}

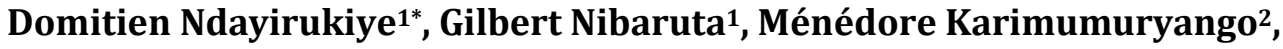 \\ Aboubacar Nibirantiza ${ }^{3}$ \\ ${ }^{1}$ Ecole Normale Supérieure, Département des Sciences Naturelles, Bujumbura, Burundi \\ ${ }^{2}$ Université du Burundi, Institut des Statistiques, Bujumbura, Burundi \\ ${ }^{3}$ Université du Burundi, Institut de Pédagogie Appliquée, Département des Mathématiques, Bujumbura, Burundi \\ Email: *domitienndayi@yahoo.fr
}

How to cite this paper: Ndayirukiye, D., Nibaruta, G., Karimumuryango, M. and Nibirantiza, A. (2019) Algebraicity of Induced Riemannian Curvature Tensor on Lightlike Warped Product Manifolds. Journal of Applied Mathematics and Physics, 7, 3132-3139.

https://doi.org/10.4236/jamp.2019.712220

Received: October 23, 2019

Accepted: December 16, 2019

Published: December 19, 2019

Copyright $\odot 2019$ by author(s) and Scientific Research Publishing Inc. This work is licensed under the Creative Commons Attribution International License (CC BY 4.0).

http://creativecommons.org/licenses/by/4.0/

\begin{abstract}
Lightlike warped product manifolds are considered in this paper. The geometry of lightlike submanifolds is difficult to study since the normal vector bundle intersects with the tangent bundle. Due to the degenerate metric, the induced connection is not metric and it follows that the Riemannian curvature tensor is not algebraic. In this situation, some basic techniques of calulus are not useable. In this paper, we consider lightlike warped product as submanifold of semi-Riemannian manifold and establish some remarkable geometric properties from which we establish some conditions on the algebraicity of the induced Riemannian curvature tensor.
\end{abstract}

\section{Keywords}

Lightlike (Sub)Manifolds, Algebraic Curvature Tensor, Total Umbilicity

\section{Introduction}

Semi-Riemannian geometry is the study of smooth manifolds with non-degenerate metric signature [1]. Semi-Riemannian geometry includes the Riemannian geometry with a positive definite metric and Lorentzian geometry which is the mathematical theory used in General Relativity.

In 1969, Bishop and O'Neill [2] introduced a new concept of warped product manifolds to construct a rich variety of manifolds with useful applications in General Relativity on the study of cosmological models and black holes. For example, it has been pointed out in [3] that some well-known exact solutions to Einstein field equations are semi-Riemannian warped products.

It is well-known that for any semi-Riemannian (warped product) manifold, 
there is a natural existence for lightlike subspaces. Thus there exists a particular case of submanifolds namely lightlike (degenerate) [4]. The geometry of lightlike submanifolds is different from the non-lightlike one and rather difficult since its normal vector bundle intersects with the tangent bundle. Due to the degenerate metric induced on a lightlike manifold, the induced connection is not metric and it follows that the Riemannian curvature tensor is not algebraic. Thus, one can not use, in the usual way, the habitual submanifold theory to define any induced object on a degenerate submanifold.

A Riemannian curvature tensor of a semi-Riemannian manifold $(M, g)$ is algebraic if it has the following symetry properties

$$
\begin{gathered}
R(X, Y, Z, W)=R(Z, W, X, Y)=-R(Y, X, Z, W) \\
R(X, Y, Z, W)+R(Y, Z, X, W)+R(Z, X, Y, W)=0 \\
\forall X, Y, Z, W \in T_{p} M .
\end{gathered}
$$

The notion of curvature is one of the central concepts of differential geometry, one could argue that is the one central on, distinguishing the geometrical core of the subject from those aspects that are analytic, algebraic, or topological [5]. Curvature also plays a key role in physics. The motion of a body in a gravitational field is determined, according to Einstein, by the curvature of space-time.

Since the whole curvature tensor is difficult to handle, the investigation usually focuses on different objects whose properties allow us to recover curvature tensor. One can associate to $\mathrm{R}$ an endomorphism on tangent bundle of a manifold [6]. In lightlike geometry, to make such study, we have to ensure that the Riemannian tensor has the algebraic proprties.

Although the lightlike geometry is difficult to study, there are important applications in Physic. In [7] the author used the warped product technique to study a problem concerning of finding a warping function such that the degenerate metric of a globally lightlike warped product manifold admits constant scalar curvature and discovered that this approach has an interplay with the static vaccum solutions of Einstein equation of general relativity.

In this paper, we examine some conditions on lightlike warped product (sub-)manifolds to admit an algebraic curvature tensor. We particularly consider single lightlike warped product (sub-)manifolds and present some technical and characterization results (Proposition 2, Proposition 3, Proposition 4). We establish algebraicity condition for the (induced) Riemannian curvature tensor on lightlike warped product submanifold (Theorm 5, Theorem 6).

\section{Basic Notions on Lightlike Geometry}

For more details see [4]. Let $(\bar{M}, \bar{g})$ be a $(m+k)$-dimensional semi-Riemannian manifold of constant index $q$ such that $1 \leq q<m+k$ and $(M, g)$ be a $m$-dimensional submanifold of $\bar{M}$. We assume that both $m$ and $k$ are $\geq 1$. At each point $p \in M$,

$$
T_{p} M^{\perp}=\left\{X \in T_{p} \bar{M}, \bar{g}_{p}(X, Y)=0, \forall Y \in T_{p} M\right\}
$$


is the normal space at $p$. In case $\bar{g}_{p}$ is non-degenerate on $T_{p} M$, both $T_{p} M$ and $T_{p} M^{\perp}$ are non-degenerate and we have $T_{p} M \cap T_{p} M^{\perp}=\{0\}$. If the mapping

$$
\operatorname{Rad}(T M): p \in M \mapsto \operatorname{Rad}\left(T_{p} M\right)=T_{p} M \cap T_{p} M^{\perp}
$$

is a smooth distribution with constant rank $r>0, M$ is said to be lightlike (or lightlike) submanifold of $\bar{M}$, with lightlikeity degree $r$. This mapping is called the radical distribution on $M$. Any complementary (and hence orthogonal) distribution of $\operatorname{Rad}(T M)$ in $T M$ is called a screen distribution. For a fixed screen distribution on $M$, the tangent bundle splits as

$$
T M=\operatorname{Rad}(T M) \oplus_{\text {orth }} S(T M) .
$$

$\oplus_{\text {orth }}$ is the orthogonal direct sum. A screen transversal vector bundle $S\left(T M^{\perp}\right)$ on $M$ is any (semi-Riemannian) complementary vector bundle of $\operatorname{Rad}(T M)$ in $T M^{\perp}$. It is obvious that both $S\left(T M^{\perp}\right)$ and $S(T M)^{\perp}$ is non-degenerate with respect to $\bar{g}$ and

$$
S\left(T M^{\perp}\right) \subset S(T M)^{\perp} .
$$

A lightlike submanifold $M$ with lightlikeity degree $r$ equipped with a screen distribution $S(T M)$ and a screen transversal vector bundle $S\left(T M^{\perp}\right)$ is denoted $\left(M, S(T M), S\left(T M^{\perp}\right)\right)$. It is said to be

1) $r$-lightlike if $r<\min (m, k)$;

2) Coisotropic if $r=k<m$ (hence $\left.S\left(T M^{\perp}\right)=\{0\}\right)$;

3) Isotropic if $r=m<k$, (hence $S(T M)=\{0\}$ );

4) Totally lightlike if $r=m=k$, (hence $S(T M)=\{0\}=S\left(T M^{\perp}\right)$ ).

For any local frame $\left\{\xi_{i}\right\}$ of $\operatorname{Rad}(T M)$, there exists a local frame $\left\{N_{i}\right\}$ of sections with values in the orthogonal complement of $S\left(T M^{\perp}\right)$ in $S(T M)^{\perp}$ such that

$$
g\left(\xi_{i}, N_{j}\right)=\delta_{i j}, g\left(N_{i}, N_{j}\right)=0
$$

and it follows that there exists a lightlike transversal vector bundle $\operatorname{ltr}(T M)$ locally spanned by $\left\{N_{i}\right\}$.

If we denote by $\operatorname{tr}(T M)$ a (not orthogonal) complementary vector bundle to $T M$ in $\left.T \bar{M}\right|_{M}$, the following relations hold

$$
\begin{gathered}
\operatorname{tr}(T M)=\operatorname{ltr}(T M) \oplus_{\text {orth }} S\left(T M^{\perp}\right), \\
\left.T \bar{M}\right|_{M}=T M \oplus \operatorname{tr}(T M)=S(T M) \oplus_{\text {orth }}(\operatorname{Rad}(T M) \oplus \operatorname{ltr}(T M)) \oplus_{\text {orth }} S\left(T M^{\perp}\right) .
\end{gathered}
$$

The Gauss and Weingarten formulas are

$$
\begin{array}{r}
\bar{\nabla}_{X} Y=\nabla_{X} Y+h(X, Y), \\
\bar{\nabla}_{X} V=-A_{V} X+\nabla_{X}^{t} V,
\end{array}
$$

$\forall X, Y \in \Gamma(T M), V \in \Gamma(\operatorname{tr}(T M))$. The components $\nabla_{X} Y$ and $-A_{V} X$ belong to $\Gamma(T M), h(X, Y)$ and $\nabla_{X}^{t} V$ to $\Gamma(\operatorname{tr}(T M)) . \nabla$ and $\nabla^{t}$ are linear connections on $T M$ and the vector bundle $\operatorname{tr}(T M)$ respectively. According to 
the decomposition (7), let $L$ and $S$ denote the projection morphisms of $\operatorname{tr}(T M)$ onto $\operatorname{ltr}(T M)$ and $S\left(T M^{\perp}\right)$ respectively, $h^{l}=L \circ h, h^{s}=S \circ h$ where $\circ$ is the composition law. $D_{X}^{l} V=L\left(\nabla_{X}^{t} V\right)$ and $D_{X}^{s} V=S\left(\nabla_{X}^{t} V\right)$. The transformations $D^{l}$ and $D^{s}$ do not define linear connections but Otsuki connections on $\operatorname{tr}(T M)$ with respect to the vector bundle morphisms $L$ and $S$. Then, $\forall X, Y \in \Gamma(T M), N \in \Gamma(\operatorname{ltr}(M))$ and $W \in \Gamma\left(S\left(T M^{\perp}\right)\right)$ we have

$$
\begin{aligned}
& \bar{\nabla}_{X} Y=\nabla_{X} Y+h^{l}(X, Y)+h^{s}(X, Y) \\
& \bar{\nabla}_{X} N=-A_{N} X+D_{X}^{l} N+D^{s}(X, N) \\
& \bar{\nabla}_{X} W=-A_{W} X+\nabla_{X}^{s} W+D^{l}(X, W) .
\end{aligned}
$$

Since $\bar{\nabla}$ is a metric connection, using (11)-(13) we have

$$
\begin{gathered}
\bar{g}\left(h^{s}(X, Y), W\right)+\bar{g}\left(Y, D^{l}(X, W)\right)=g\left(A_{W} X, Y\right) \\
\bar{g}\left(D^{s}(X, N), W\right)=\bar{g}\left(N, A_{W} X\right) .
\end{gathered}
$$

Let $P$ the projection morphism of $T M$ onto $S(T M)$. Using the decomposition (5) we get

$$
\begin{gathered}
\nabla_{X} Y=\nabla_{X}^{*} P Y+h^{*}(X, P Y) \\
\nabla_{X} \xi=-A_{\xi}^{*} X+\nabla_{X}^{* t} \xi
\end{gathered}
$$

$\forall X, Y \in \Gamma(T M), \xi \in \Gamma(\operatorname{Rad}(T M))$ and $\nabla^{*}$ is a metric connection on $S(T M)$.

It follows from (16) and (17) that

$$
\begin{gathered}
\bar{g}\left(h^{l}(X, P Y)\right)=g\left(A_{\xi}^{*} X, P Y\right) \\
\bar{g}\left(h^{*}(X, P Y), N\right)=g\left(A_{N} X, P Y\right) \\
\bar{g}\left(h^{l}(X, \xi), \xi\right)=0, A_{\xi}^{*} \xi=0 .
\end{gathered}
$$

Let $\bar{R}$ and $R$ denote the Riemannian curvature tensors on $\bar{M}$ and $M$ respectively. The Gauss equation is given by

$$
\begin{aligned}
\bar{R}(X, Y) Z= & R(X, Y) Z+A_{h^{l}(X, Z)} Y-A_{h^{l}(Y, Z)} X+A_{h^{s}(X, Z)} Y \\
& -A_{h^{s}(X, Z)} X+\left(\nabla_{X} h^{l}\right)(Y, Z)-\left(\nabla_{Y} h^{l}\right)(X, Z) \\
& +D^{l}\left(X, h^{s}(Y, Z)\right)-D^{l}\left(Y, h^{s}(X, Z)\right)+\left(\nabla_{X} h^{s}\right)(Y, Z) \\
& -\left(\nabla_{Y} h^{s}\right)(X, Z)+D^{s}\left(X, h^{l}(Y, Z)\right)-D^{s}\left(Y, h^{s}(X, Z)\right)
\end{aligned}
$$

$\forall X, Y, Z, U \in \Gamma(T M)$. Therefore

$$
\begin{aligned}
& \bar{R}(X, Y, Z, P U) \\
= & R(X, Y, Z, P U)+\bar{g}\left(h^{*}(Y, P U), h^{l}(X, Z)\right)-\bar{g}\left(h^{*}(X, P U), h^{l}(Y, Z)\right) \\
& +\bar{g}\left(h^{s}(Y, P U), h^{s}(X, Z)\right)-\bar{g}\left(h^{s}(X, P U), h^{s}(Y, Z)\right) .
\end{aligned}
$$

Definition 2.1. [8] A lightlike submanifold $(M, g)$ of a semi-Riemannian manifold $(\bar{M}, \bar{g})$ is totally umbilical in $\bar{M}$ if there is a smooth transversal vector field $\boldsymbol{H} \in \Gamma(\operatorname{tr}(T M))$ on $M$ called the transversal curvature vector field 
of $M$ such that, for all $X, Y \in \Gamma(T M)$

$$
h(X, Y)=g(X, Y) \boldsymbol{H} .
$$

Using (9) and (11) its is easy to see that $M$ is totally umbilical if and only if on each coordinate neighbourhood $\mathcal{U}$ there exist smooth vector fields $H^{l} \in \Gamma(\operatorname{ltr}(T M))$ and $H^{s} \in \Gamma\left(S\left(T M^{\perp}\right)\right)$ such that

$$
\begin{gathered}
h^{l}(X, Y)=g(X, Y) H^{l}, D^{l}(X, W)=0 \\
h^{s}(X, Y)=g(X, Y) H^{s}, \forall X, Y \in \Gamma(T M), W \in \Gamma\left(S\left(T M^{\perp}\right)\right) .
\end{gathered}
$$

Definition 2.2. [8] Let $(M, g)$ be a r-lightlike (i.e. $r<\min \{m, k\})$ or a coisotropic $m$-dimensional submanifold of a $(m+k)$-dimensional semi-Riemannian manifold $(\bar{M}, \bar{g})$. We say that the screen distribution $S(T M)$ is totally umbilical if for any section $N$ of $\operatorname{ltr}(T M)$ on a coordinate neighbourhood $\mathcal{U} \subset M$, there exists a smooth function $\lambda$ on $\mathcal{U}$ such that

$$
\bar{g}\left(h^{*}(X, P Y), N\right)=\lambda g(X, P Y), \forall X, Y \in \Gamma\left(\left.T M\right|_{\mathcal{U}}\right) \text {. }
$$

Definition 2.3. A coisotropic submanifold $(M, g)$ of a semi-Riemannian manifold $(\bar{M}, \bar{g})$ is screen locally conformal if the local second fundamental forms of the screen distribution $S(T M)$ are related with the local second fundamental form of $M$ as follows.

$$
h_{i}^{*}(X, P Y)=\phi_{i} h_{i}^{l}(X, P Y), \forall X, Y \in \Gamma(T M)
$$

where $\phi_{i}$ is a conformal smooth function in a coordinate neighbourhood $\mathcal{U}$ in $M$. In particular, we say that $M$ is sreen homothetic if $\phi_{i}$ is a non-zero constant.

Definition 2.4. Let $\left(M_{0}, g_{0}\right)$ and $\left(M_{i}, g_{i}\right)(i=1,2, \cdots, l)$ be semi-Riemannian manifolds and $\left.\rho_{i}: M_{0} \rightarrow\right] 0,+\infty[$ be positive smooth functions. The multiply warped product $M=M_{0} \times{ }_{\rho_{1}} M_{1} \times{ }_{\rho_{2}} M_{2} \times \cdots \times{ }_{\rho_{l}} M_{l}$ is the product manifold $M_{0} \times M_{1} \times M_{2} \times \cdots \times M_{l}$ furnished with the metric tensor

$$
g=\pi_{0}^{*}\left(g_{0}\right)+\sum_{i=1}^{l}\left(\rho_{i} \circ \pi_{0}\right)^{2} \pi_{i}^{*}\left(g_{i}\right)
$$

where $\pi_{0}: M \rightarrow M_{0}, \pi_{i}: M \rightarrow M_{i}, i=1, \cdots, l$ are the projection morphisms. The functions $\rho_{i}$ are called the warping functions and $\left(M_{0}, g_{0}\right)$ the base manifold of the multiply warped product. Each $\left(M_{i}, g_{i}\right), i=1, \cdots, l$ is called a fiber manifold.

- If $l=1$ then we obtain a singly warped product.

- If $\rho_{i}=1$ for $i=1, \cdots, l$ then we have a muliple product manifold.

- If all $\left(M_{i}, g_{i}\right), i=0,1, \cdots, l$ are Riemanniann manifolds then $(M, g)$ is also a Riemannian multiply warped product manifold. $(M, g)$ is Lorentzian multiply warped product if $\left(M_{i}, g_{i}\right), i=1, \cdots, l$ are Riemannian and either $\left(M_{0}, g_{0}\right)$ is Lorentzian or a one-dimensional manifold with a negative definite metric $-d t^{2}$.

- $(M, g)$ is lightlike (lightlike) with lightlikeity degree $r$ if $\left(M_{0}, g_{0}\right)$ is degenerate with $\operatorname{Rad}\left(T M_{0}\right)$ of rank $r . \operatorname{Rad}(T M)$ still has rank $r$ and all screen 
structure on $M$ has dimension $s_{0}+\sum_{i=1}^{m} \operatorname{dim}\left(M_{i}\right)$ where $s_{0}$ is the dimension of any screen structure on $M_{0}$.

For a singly warped product, we have the following:

Proposition 1. [1] On $\left(N_{1} \times{ }_{\rho} N_{2}, g\right)$, if $X, Y \in \Gamma\left(T N_{1}\right) ; V, W \in \Gamma\left(T N_{2}\right)$, then,

1) $\nabla_{X} Y \in \Gamma\left(T N_{1}\right)$ is the lift of $\nabla_{X}^{1} Y$;

2) $\nabla_{X} V=\nabla_{V} X=\frac{X(\rho)}{\rho} V$;

3) $\left(\nabla_{V} W\right)_{\mathcal{V}}$ is the lift of $\nabla_{V}^{2} W$;

4) $\left(\nabla_{V} W\right)_{\mathcal{H}}=\alpha(V, W)=-\frac{\langle V, W\rangle}{\rho} \operatorname{grad} \rho$.

From the previous proposition, one can see that

$$
g\left(X_{1}, X_{2}\right)=0, \forall X_{1} \in \Gamma\left(T N_{1}\right), X_{2} \in \Gamma\left(T N_{2}\right) .
$$

Definition 2.5. A lightlike warped product submanifold $M=N_{1} \times_{\rho} N_{2}$ of a semi-Riemannian manifold $\bar{M}$ is called mixed totally geodesic if $h\left(X_{1}, Y_{2}\right)=0$ for any $X_{1} \in \Gamma\left(T N_{1}\right)$ and $Y_{2} \in \Gamma\left(T N_{2}\right)$.

\section{Our Main Results}

In the following, we consider a lightlike warped product $\left(M=N_{1} \times_{\rho} N_{2}, g_{1} \oplus \rho^{2} g_{2}\right)$ isometrically immersed in a semi-Riemannian manifold $(\bar{M}, \bar{g})$.

Proposition 2. Let $f$ be a coisotropic isometric immersion of a warped product $\left(M=N_{1} \times_{\rho} N_{2}, g_{1} \oplus \rho^{2} g_{2}\right)$ into a semi-Riemannian manifold $(\bar{M}, \bar{g})$ with the first factor $N_{1}$ totally degenerate. Then $f$ is a totally umbilical isometric immersion.

Proof. In case of coisotropic submanifold we have

$$
\bar{\nabla}_{X} Y=\nabla_{X} Y+h^{l}(X, Y), \forall X, Y \in \Gamma(T M) \text { where } h^{l}(X, Y)=\sum_{i=1}^{r} h_{i}^{l}(X, Y) N_{i} .
$$

Thus

$$
h_{i}^{l}(X, Y)=\bar{g}\left(\bar{\nabla}_{X} Y, \xi_{i}\right)=-g\left(Y, \nabla_{X} \xi_{i}\right)=-\frac{\xi_{i}(\rho)}{\rho} g(X, Y) .
$$

Then

$$
h^{l}(X, Y)=-\sum_{i=1}^{r} \frac{\xi_{i}(\rho)}{\rho} g(X, Y) N_{i}
$$

that is $h^{l}(X, Y)=g(X, Y) H^{l}$ where $H^{l}=-\sum_{i=1}^{r} \frac{\xi_{i}(\rho)}{\rho} N_{i}$.

Proposition 3. Any totally umbilical lightlike warped product submanifold of a semi-Riemannian manifold is mixed totally geodesic.

Proof. From the expressions (27) and (2.1), we have $h^{l}\left(X_{1}, Y_{2}\right)=0$ and $h^{s}\left(X_{1}, Y_{2}\right)=0$ i.e $h\left(X_{1}, Y_{2}\right)=0, \forall X_{1} \in \Gamma\left(T N_{1}\right), X_{2} \in \Gamma\left(T N_{2}\right)$.

Proposition 4. Let $M=N_{1} \times_{\rho} N_{2}$ be a lightlike warped product submanifold 
of a semi-Riemannian manifold $(\bar{M}, \bar{g})$ with $N_{1}$ totally degenerate. Then $\forall \xi \in \Gamma(\operatorname{Rad}(T M)), \quad Y \in \Gamma(S(T M)), W \in \Gamma\left(S\left(T M^{\perp}\right)\right)$ we have

1) $h^{l}(\xi, Y)=0$;

2) $h^{*}(\xi, Y)=0$;

3) $A_{N} \xi \in \Gamma(\operatorname{Rad}(T M))$;

4) $D^{l}(\xi, W)=0$;

5) $\bar{\nabla}_{\xi} N \in \Gamma(\operatorname{tr}(T M))$.

Proof. Let $\xi_{1}, \xi_{2} \in \Gamma(\operatorname{Rad}(T M)), Y \in \Gamma(S(T M))$. From (11) and proposition 1 we have

$$
\bar{g}\left(h^{l}\left(\xi_{1}, Y\right), \xi_{2}\right)=\bar{g}\left(\bar{\nabla}_{\xi_{1}} Y, \xi_{2}\right)=-\bar{g}\left(Y, \bar{\nabla}_{\xi_{1}} \xi_{2}\right)=-g\left(Y, \nabla_{\xi_{1}} \xi_{2}\right)=0 .
$$

From (16) we have

$$
\bar{g}\left(h^{*}(\xi, Y), N\right)=\bar{g}\left(\nabla_{\xi} Y, N\right)=\frac{\xi(\rho)}{\rho} \bar{g}(Y, N)=0 .
$$

From (19) and (30) we have $A_{N} \xi \in \Gamma(\operatorname{Rad}(T M))$.

Let $\xi_{1}, \xi_{2} \in \Gamma(\operatorname{Rad}(T M)), W \in \Gamma\left(S\left(T M^{\perp}\right)\right)$. From (13) we have

$$
\bar{g}\left(D^{l}\left(\xi_{1}, W\right), \xi_{2}\right)=\bar{g}\left(\bar{\nabla}_{\xi_{1}} W, \xi_{2}\right)=-\bar{g}\left(W, \bar{\nabla}_{\xi_{1}} \xi_{2}\right)=-\bar{g}\left(W, \nabla_{\xi_{1}} \xi_{2}\right)=0 .
$$

Using (11), (16), (29) and (30) we have $\bar{\nabla}_{\xi} Y=\nabla_{\xi}^{*} Y+h^{s}(\xi, Y)$. Then $\bar{g}\left(\bar{\nabla}_{\xi} Y, N\right)=0$ and since $\bar{\nabla}$ is a metric connexion we have

$$
\bar{g}\left(Y, \bar{\nabla}_{\xi} N\right)=0, \forall Y_{2} \in \Gamma\left(T N_{2}\right), \xi \in \Gamma(\operatorname{Rad}(T M)), \nabla_{\xi} N \in \Gamma(\operatorname{tr}(T M)) .
$$

Moreover $\bar{\nabla}_{\xi} N \in \Gamma(\operatorname{tr}(T M))$.

We give the following result on the algebraic properties of the induced Riemannian tensor on lightlike warped product with the first factor totally degenerate.

Theorem 5. Let $N_{1} \times{ }_{\rho} N_{2}$ be a lightlike submanifold of a semi-Riemannian manifold $(\bar{M}, \bar{g})$ equiped by an induced lightlike warperd product metric $g_{1} \oplus \rho^{2} g_{2}$ with the first factor $N_{1}$ totally degenerate. Then the induced Riemannian curvature is an algebraic tensor.

Proof. The result hold from Theorem 3.2 in [9] and proposition 4.

In case of coisotropic warped product of a semi-Riemannian manifold with constant sectional curvature which is conformal screen, we establish the following.

Theorem 6. Let $f:\left(M=N_{1} \times_{\rho} N_{2}, g_{1} \oplus \rho^{2} g_{2}\right) \mapsto(\bar{M}(c), \bar{g})$ be a coisotropic isometric immersion of a lightlike warped product into a semi-Riemannian manifold which is a space form such that the lightlike warped product $M$ is conformal screen. Then the induced Riemannian curvature $R$ is an algebraic curvature tensor.

Proof. Since $\bar{M}$ has constant sectional curvature $c$, we have $\bar{R}(X, Y) Z=g(Y, Z) X-g(X, Z) Y \quad([1]$, p. 80). From (22), $\forall X, Y, Z, T \in \Gamma(T M)$, taking account $M$ is conformal screen coisotropic manifold, we have

$$
\begin{aligned}
R(X, Y, Z, P T)= & c\{g(Y, Z) g(X, P T)-g(X, Z) g(Y, P T)\} \\
& +\sum_{i=1}^{r} r \phi_{i}\left\{h_{i}^{l}(X, P T) h_{i}^{l}(Y, Z)-h_{i}^{l}(Y, P T) h_{i}^{l}(X, Z)\right\} .
\end{aligned}
$$


It is then obvious that $R(X, Y, Z, P T)$ holds (1) and (2). Consider now Proposition (4), $\forall Y, Z \in \Gamma(T M), \xi \in \Gamma(\operatorname{Rad}(T M))$, we have

$$
\begin{gathered}
R(X, Y, Z, \xi)=-R(Y, X, Z, \xi)=0, \\
R(Z, \xi, X, Y)=\bar{R}(Z, \xi, X, Y)=-\bar{R}(X, Y, \xi, Z)=0
\end{gathered}
$$

and we infer $R(X, Y, Z, \xi)+R(Y, Z, X, \xi)+R(Z, X, Y, \xi)=0$ to conclude.

\section{Conclusion and Suggestions}

The algebraicity conditions of the induced Riemannian curvature tensor have been explored in this paper. Some remarkable geometric properties of lightlike warped product submanifolds have been given. From the above results, one can see that the induced Riemannian curvature tensor on lightlike warped product submanifolds with totally null first factor is an algebraic curvator tensor. In the future, we will be studying Osserman conditions on lightlike warped product manifolds.

\section{Conflicts of Interest}

The authors declare no conflicts of interest regarding the publication of this paper.

\section{References}

[1] O’Neill, B. (1983) Semi-Riemannian Geometry with Applications to Relativity. Academic Press, New York, 323-325.

[2] Bishop, R.L. and O’Neil, B. (1969) Manifolds of Negative Curvature. Transactions of the American Mathematical Society, 145, 1-49. https://doi.org/10.2307/1995057

[3] Beem, J.K., Ehrich, P.E. and Easley, K.L. (1996) Global Lorentzian Geometry 2nd Edition, Markel-Deccer Inc., New York, 1-345.

[4] Duggal, K.L. and Bejancu, A. (1996) Lightlike Submanifolds of Semi-Riemannian Manifolds and Applications. Kluwer Academic Publishers, Amsterdam, 308. https://doi.org/10.1007/978-94-017-2089-2

[5] Uwe, A. (1985) Lower Curvature Bounds, Toponogov's Theorem, and Bounded Topology. Annales scientifiques de I École Normale Supérieure, 18, 651-670. https://doi.org/10.24033/asens.1499

[6] Gilkey, P.B. (2002) Geometric Properties of Natural Operators Defined by the Riemann Curvature Tensor. World Scientific, Singapore.

https://doi.org/10.1142/9789812799692

[7] Duggal, K.L. (2002) Constant Scalar Curvature and Warped Product Globally Lightlike Manifolds. Journal of Geometry and Physics, 43, 327-340. https://doi.org/10.1016/S0393-0440(02)00032-3

[8] Duggal, K.L. and Jin, D.H. (2003) Totally Umbilical Lightlike Submanifolds. Kodai Mathematical Journal, 26, 49-68. https://doi.org/10.2996/kmj/1050496648

[9] Atindogbe, C., Lungiambudila, O. and Tossa, J. (2011) Ligthlike Osserman Submanifolds of Semi-Riemannian Manifolds. Afrika Matematika, 22, 129-151. https://doi.org/10.1007/s13370-011-0015-0 